\title{
Re: Community-Based Outcomes of Open Versus Robot-Assisted Radical Prostatectomy
}

\begin{abstract}
Herlemann $\mathrm{A}^{1}$, Cowan JE², Carroll PR², Cooperberg $\mathrm{MR}^{3}$
'University of California San Francisco, Helen Diller Family Comprehensive Cancer Center, Department of Urology, San Francisco, CA, USA; Ludwig-Maximilians-University of Munich, Department of Urology, Munich, Germany ${ }^{2}$ University of California San Francisco, Helen Diller Family Comprehensive Cancer Center, Department of Urology, San Francisco, CA, USA ${ }^{3}$ University of California San Francisco, Helen Diller Family Comprehensive Cancer Center, Department of Urology, San Francisco, CA, USA; University of California San Francisco, Helen Diller Family Comprehensive Cancer Center, Department of Epidemiology and Biostatistics, San Francisco, CA, USA
\end{abstract}

Eur Urol 2018;73:215-223. doi: 10.1016/j.eururo.2017.04.027.

\section{EDITORIAL COMMENT}

Robot-assisted radical prostatectomy (RARP) has been increasingly used as a minimally invasive technique for the surgical treatment of prostate cancer (PCa) in both academic- and community-based centers. Numerous retrospective studies with weak level of evidence have suggested superiority in surgical, oncologic and functional outcomes of RARP over open radical prostatectomy (ORP). Fewer have suggested otherwise. The majority of such studies have been based on data derived from high-volume academic medical centers. Only one prospective randomized study was published recently which demonstrated similar functional outcomes at 12 weeks for RARP and ORP (1). The authors of this study assessed surgical outcomes and urinary and sexual quality of life in patients undergoing ORP versus RARP. The study included patients enrolled in Cancer of the Prostate Strategic Urologic Research Endeavor (CaPSURE) who underwent radical prostatectomy between 2004 and 2016. CaPSURE is a mostly community-based large prospective PCa registry in the USA. Among 1892 men, 1137 underwent ORP, and 755 RARP. Surgical margin rates and 5-year biochemical recurrence-free survival rates were similar in both groups. Urinary and sexual quality of life outcomes were similar beyond 12 months. This study underlines the fact that, there is yet no high-level evidence suggesting superiority of RARP or ORP over the other for oncologic or quality of life outcomes.

\section{Reference}

1. Yaxley JW, Coughlin GD, Chambers SK, Occhipinti S, Samaratunga H, Zajdlewicz L, Dunglison N, Carter R, Williams S, Payton DJ, Perry-Keene J, Lavin MF, Gardiner RA. Robot-assisted laparoscopic prostatectomy versus open radical retropubic prostatectomy: early outcomes from a randomised controlled phase 3 study. Lancet 2016;388:1057-1066.

Özgür Yaycıoğlu, MD

\footnotetext{
- Copyright 2018 by the Association of Urological Surgery / Journal of Urological Surgery published by Galenos Publishing House.
} 